\title{
Non-palpable breast lesions: out-patient needle localization and biopsy
}

\author{
James Smith-Behn and A. Ghani \\ Division of Surgery, Youngstown Hospital Association and Northeastern Ohio Universities, College of Medicine, \\ 345 Oak Hill Drive, Youngstown, Ohio 44503, USA.
}

\begin{abstract}
Summary: One hundred and nine patients undergoing out-patient needle localization and biopsy of non-palpable breast lesions were studied. Six patients with carcinoma were detected, 5 of them qualifying as minimal carcinoma. Thirty five patients with proliferative fibrocystic breast disease in need of careful follow-up were identified. While the number of patients with carcinoma was low, the early stage of their disease justifies continued use of the procedure. Doing the procedure on an out-patient basis has been shown by others to provide a substantial reduction in cost. Our results suggest that the out-patient setting does not affect the morbidity or mortality of the procedure.
\end{abstract}

\section{Introduction}

With the advent of mammography as a routine method of screening for breast carcinoma, an increasing number of patients are undergoing breast biopsy. The patient with a mammographic abnormality and a non-palpable mass presents a challenge to the surgeon. Precise isolation and excision of the suspicious lesion becomes very difficult. Pre-operative localization of these areas has minimized the volume of tissue removed from the questioned area, thereby allowing a better cosmetic result in patients with benign breast disease, but assuring removal of the suspicious area. This report describes our experience at the Western Reserve Care System with out-patient needle localization and biopsy of non-palpable breast lesions, and compares our results with those obtained at other institutions.

\section{Patients and methods}

All patients undergoing needle localization of nonpalpable breast lesions between February 1985 and January 1986 were studied. The factors evaluated were the patient's age, the mammographic abnormality which prompted biopsy, the histology of the tissue removed and subsequent staging and management of patients found to have carcinoma.

Patients came to the hospital on the morning of surgery and were taken to the radiology department for needle localization under local anaesthesia. A

Correspondence: J. Smith-Behn, M.D.

Accepted: 31 July 1986
Kopans needle (Cook Industries, Bloomington, Indiana) was used in most of our patients; however, for lesions close to the chest wall, an angiocath (no. 2812, 14 gauge, 2": Deseret Pharmaceutical, Sandy, Utah) was used. A drop of methylene blue was injected through the angiocath at the end of the procedure. A lesion was considered to be adequately localized when the marker was within $1 \mathrm{~cm}$ of the lesion. Patients were then taken to the operating room and the lesion excised under general anaesthesia. All specimens were returned to the radiology department for mammographic identification, and the tissue was then submitted to the pathologist for examination. Patients were discharged several hours after completion of the procedure.

\section{Results}

A total of 109 patients underwent needle localization of the non-palpable lesions. All but one of the procedures were done on an out-patient basis. Patients' ages ranged from 38 to 83 years with a mean of 57 years. The mammograms reviewed showed that $35 \%(38 / 109)$ of the patients had microcalcifications, $58 \%(63 / 109)$ a mass, and $7 \%(8 / 109)$ both a mass and microcalcifications. Six patients $(5.5 \%)$ were found to have carcinoma; 4 of them infiltrating ductal, one infiltrating ductal and lobular, and one infiltrating adenocarcinoma. Three of the carcinomas occurred in patients with microcalcifications and 3 in patients described as having masses. One patient $(0.9 \%)$ had a benign cystosarcoma phylloides. Thirty five patients

(C) The Fellowship of Postgraduate Medicine, 1987 
$(32 \%)$ were found to have proliferative fibrocystic breast disease, and 67 patients $(61 \%)$ had nonproliferative fibrocystic breast disease.

The patients who were found to have carcinoma all had T1 lesions. Five of them could be classified as minimal carcinoma. Four of the patients underwent modified radical mastectomy as definitive treatment while 2 opted for axillary node dissection and radiation therapy. Only 1 patient was found to have positive axillary nodes.

\section{Discussion}

According to Walker et al. ${ }^{1}$ breast cancer is the leading cause of death from malignancy in American women today. While patients with early lesions have a good prognosis, those with more advanced disease continue to have a poor prognosis despite advances in chemotherapy. The emphasis in the treatment of breast carcinoma has thus shifted to the detection of very early lesions. Hartmann's ${ }^{2}$ update on minimal breast carcinoma provides evidence that lesions under $1 \mathrm{~cm}$ have an excellent prognosis. Unfortunately lesions this size are, for the most part, non-palpable.

Kopans et al. $^{3}$ have shown that mammography provides a means of detecting these clinically occult lesions with a minimum of radiation exposure. In the past, clinical localization of the detected area required multiple biopsies or, at times, removal of a whole quadrant of breast to remove succesfully the suspicious area. For those patients found to have benign breast disease (the majority according to Meyer et al. ${ }^{4}$ ) the results were often cosmetically unacceptable. When pre-operative needle localization is utilized, the lesion may be excised in a precise fashion with minimal damage to the surrounding tissue. Pitzen et al. ${ }^{5}$ have emphasized the need to obtain xerography of the specimen after biopsy to insure removal of the proper area.

The yield of carcinoma in non-palpable lesions varies from 9 to $42 \% .^{1,4,5,6,7}$ Patients with microcalcifications and a mass seem to have a higher incidence

\section{References}

1. Walker, H., Delaney, J. \& Gedgaudas, E. Locating nonpalpable breast lesions for the surgeon: state of the art. Minn Med 1985, 68: 437-439.

2. Hartman, W.H. Minimal breast cancer: An update. Cancer Suppl 1984, 54: 681-684.

3. Kopans, D., Meyer, J. \& Sadowsky, N. Breast imaging. In Medical Progress. N Engl J Med 1984, 310: 960-967.

4. Meyer, J., Kopans, D., Stomper, P. \& Lindfors, S. Occult breast abnormalities: percutaneous preoperative needle localization. Radiology 1984, 150: 335-337. of carcinoma than those with only a mass ( $36 v s 24 \%$ ).

The number of cancers detected in our study is low when compared to other reported series. While we cannot offer a full explanation for this, it may be due in $\varsigma$ part to the small number of patients in our study group $\overrightarrow{\vec{\rho}}$ with microcalcifications and a mass seen on mam- mography. It may also represent a reluctance on the $\frac{C}{0}$ part of our radiologists to call a mammographic $\overline{\bar{\omega}}$ abnormality benign. Bigelow et al. ${ }^{6}$ have shown that $\vec{\nabla}$ $4.5 \%$ of mammographically benign appearing lesions $\varrho$ will turn out to be carcinomas. Most of the malignan- $ळ$ cies detected, however, qualify as minimal carcinomas $\overrightarrow{0}$ and therefore have an excellent prognosis. In addition, $\overrightarrow{-}$ 35 patients with proliferative fibrocystic breast disease $\omega$ were found. These patients are at high risk for the $\frac{}{0}$ development of carcinoma and will have to be followed very closely.

Homer et al. ${ }^{7}$ have demonstrated a 68 to $80 \% \stackrel{\text { ڤ }}{\omega}$ reduction in cost when breast biopsies are done on an $\omega$ out-patient basis. All but one of our patients were done in this fashion, and none of them required $\vec{v}$ admission for complications arising from the 을 procedure. This offers a substantial reduction in cost for the procedure without increasing the morbidity or mortality.

\section{Conclusions}

The present series has a lower cancer to biopsy ra than other reported series. The cause for this or the higher rates of other reported series is undetermined. A significant number of patients in need of careful $\cong$ follow-up was identified. In addition, all procedures $\Phi$ were done on an out-patient basis, thereby achieving a $\underset{\vec{P}}{\overrightarrow{2}}$ significant reduction in cost but without increasing the morbidity or mortality.

We continue to advocate mammography as a screening procedure for early diagnosis of breast carcinoma, but encourage research into interpretaion 3 of the studies and better techniques for surgical localization and excision.

5. Pitzen, R., Urdaneta, L. \& Al-Jurf, T. Specimen N zeroradiography after needle localization and biopsy of $\mathrm{N}$ non-calcified, non-palpable lesions. Am Surg 1985, 51: 50-57.

6. Bigelow, R., Smith, R., Goodman, P. \& Wilson, F. Needle localization of non-palpable breast masses. Arch Surg 1985, 120: 565-569.

7. Homer, M., Smith, T.\& Marchant, D. Out-patient needle $\mathscr{\mathscr { S }}$ localization of non-palpable breast lesions. JAMA 1984, 252: $2452-2454$. 